\title{
Demonstration of ripple-based index for predicting fast-scale instability in switching power converters
}

\author{
E. Rodr'guez and E.Alarcón \\ Department of Electronic Engineering \\ Technical University of Catalunya \\ 08034 Barcelona, Spain. \\ Email: enricrv,ealarcon@eel.upc.edu
}

\author{
A. El Aroudi \\ Department of Electronics Electrical, \\ Engineering and Automatic Control \\ Universitat Rovira i Virgili \\ 43007 Tarragona, Spain \\ Email: abdelali.elaroudi@urv.cat
}

\begin{abstract}
In this paper a simplified model based on the exact discrete-time map of a buck switching power converter with proportional control, which captures all its dynamics, allows deriving a closed-form stability condition for predicting fast-scale instability boundary. This condition analytically demonstrates the validity of the recently proposed ripple-based index to predict fast-scale period-doubling, hitherto based on an a priori hypothesis and simulation validation, thereby demonstrating the use of the ripple index as a design-oriented tool. The equivalence of the ripple index to the condition derived from the discrete-time map endorses its use as a means to characterize the complete design space against fast-scale instabilities.
\end{abstract}

\section{INTRODUCTION}

Switching power converters, due to their switching nonlinear nature, are prone to exhibit diverse types of instabilities. Accordingly, a notable effort has been devoted to modeling their complex behavior, mainly focusing on predicting the occurrence of such different instabilities within the multidimensional parameter design space. The most well established method to predict instabilities is the average model [1]. Despite their advantageous design-oriented circuit-based perspective, average models cannot intrinsically predict all types of instability, particularly fast-scale period-doubling instabilities that eventually end up in chaotic modes. Average modeling has hitherto yielded appropriate results to carry out stability analysis for slow-dynamics. However, in recent years, next-generation power management applications which require further miniaturization and bandwidth extension requires reducing the ratio of switching frequencies and natural converter dynamics, together with considering alternative topologies and control methods, which results in the fact that fast-scale instabilities are more prone to be exhibited. In order to take into account the switching action in DC-DC converters and to capture the complete dynamics, discrete-time recurrent maps have been widely explored, not only in basic converter topologies [2]-[6] but also in more recently proposed converter topologies such as multilevel [7] or interleaved [8]. However, such modeling approach lacks a design-perspective, and requires sophisticated mathematical tools.

With the aim of deriving a design-oriented model to predict fast-scale instabilities, in [9] a ripple-based index was proposed, which complements average models. This unidimensional index is based on describing the ripple at the modulator input relative to the modulator ramp (Eq. 1), and compresses into a single index $\rho$ all the converter design space parameters, of different nature, such as converter specifications $\left(V_{g}, V_{\text {ref }}\right.$ or duty cycle $\left.(D)\right)$, reactive components $(L, C)$, control configuration $\left(k_{p}\right)$ and modulator $\left(V_{m}, f_{s}\right)$.

The model, also validated in buck and boost topologies [10], is based on the hypothesis that when the ripple index achieves a critical value $\rho_{\text {critical }}$, which mainly depends on the duty cycle, fast-scale bifurcation is exhibited. The condition for avoiding stability is, therefore:

$$
\rho=\frac{k_{p}}{V_{m}} \Delta v_{c}<\rho_{\text {critical }}
$$

The aforementioned ripple approach has been validated indepth for the complete design space, matching prediction to actual characterized and experimental behavior. However, the method is based on a starting engineering hypothesis and thus lacks a solid mathematical theory.

This paper presents the use of a simplified model based on discrete-time recurrent maps in order to obtain simplified conditions of stability and match them with the ripple-based instability boundary prediction. Section II reviews discretetime maps for a basic buck converter with proportional gain control law. In the third section, discrete-time maps are simplified using a circuit-theoretical perspective, and accordingly a closed form expression for fast-scale instability prediction is obtained. Such expression is compared to the one derived from the ripple-based approach and both are used to characterize the instability boundaries within the converter design space.

\section{DISCRETE-MAPS}

The section reviews Discrete-Time modeling and prediction of instabilities as a starting point for comparison in the following section. As a simple but representative case, a buck converter with proportional feedback (Fig. 1), has been chosen.

The stability analysis methodology based on discrete-time map is based on constructing a model, so-called Poincaré map, by sampling the dynamics of the converter at each switching instant. For a buck converter operating in continuous conduction mode, two circuit configurations occur with the 


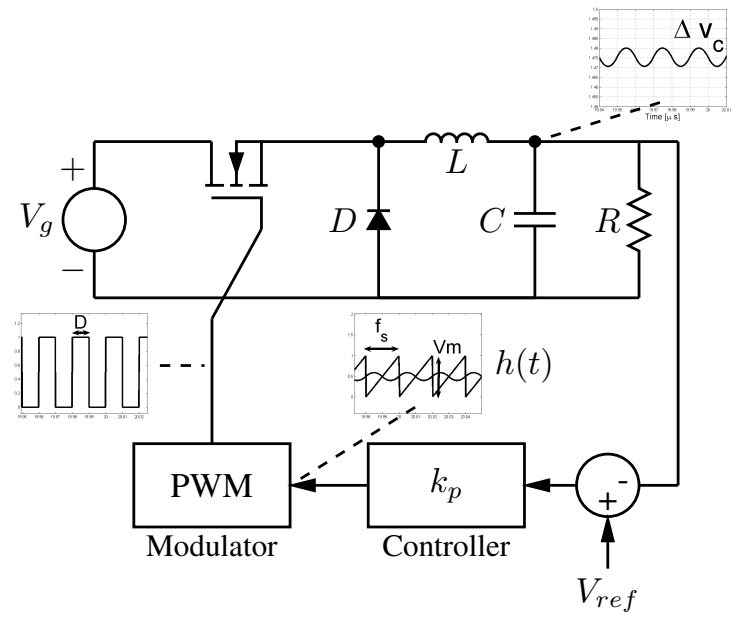

Figure 1. Buck converter with proportional feedback gain and PWM modulator

following associated linear dynamics:

$$
\begin{gathered}
\dot{x}=A_{1} x+B_{1} \text { for } t \quad\left[n T, n T+d_{n} T\right] \\
\dot{x}=A_{2} x+B_{2} \text { for } \mathrm{t} \quad\left[\left(n T+d_{n} T,(n+1) T\right]\right.
\end{gathered}
$$

where $d_{n} T$ is the time interval during which the input voltage is applied to the output $L C$ filter and $d_{n}$ is the duty cycle corresponding to cycle $[n T,(n+1) T]$.

The discrete map relates the state variables $x_{n}$ at the beginning of the cycle to $x_{n+1}$ at the end of the same cycle. For the case of a buck circuit topology, state variables are the capacitor voltage $v_{C}$ and inductor current $i_{L}$. It can be demonstrated that the map that relates both samples could be expressed as [11]:

$$
\begin{aligned}
& P\left(x_{n}\right)=X_{e q, 2}+ \\
& \quad+\phi_{2}\left(\begin{array}{ll}
T & \left.d_{n} T\right)\left(X_{e q, 1}+\phi_{1}\left(d_{n} T\right)\left(x_{n} \quad X_{e q, 1}\right) \quad X_{e q, 2}\right)(2)
\end{array}\right.
\end{aligned}
$$

Where $X_{e q, i}=A_{i}^{-1} B_{i}$ is the equilibrium point of the linear configuration $i\left(\begin{array}{ll}i & 1,2\end{array}\right)$ and $\phi_{i}\left(t_{i}\right)=e^{A_{i} t_{i}}$.

Additionally, the switching surface, which depends upon the controller law (proportional voltage gain, $k_{p}$ ) and the modulator (PWM with saw-tooth $h(t))$ can be expressed as:

$$
\sigma(t)=\mathbf{K}\left[X_{e q, 1}+\phi_{1}\left(d_{n} T\right)\left(x_{n} \quad X_{e q, 1}\right)\right] \quad h(t)
$$

where $\mathbf{K}=\left(\begin{array}{ll}k_{p} & 0\end{array}\right)$. The stability analysis is carried out by studying the local behavior of the map in the vicinity of steady-state $\left(x^{*}\right)$, thereby extracting a Jacobian matrix $D P$, the eigenvalues of which describe the amount of expansion and contraction:

$$
D P=\frac{\delta P}{\delta x_{n}} \quad \frac{\delta P}{\delta d_{n}}\left(\frac{\delta \sigma}{\delta d_{n}}\right)^{-1} \frac{\delta \sigma}{\delta x_{n}}
$$

By considering equations (2) and (3), each term of (4) can be described as:

\footnotetext{
$\frac{\delta P}{\delta x_{n}} \quad=\phi_{1}(D T) \phi_{2}(T \quad D T)$ where it is assumed that in steady state $d_{n}=D$
}
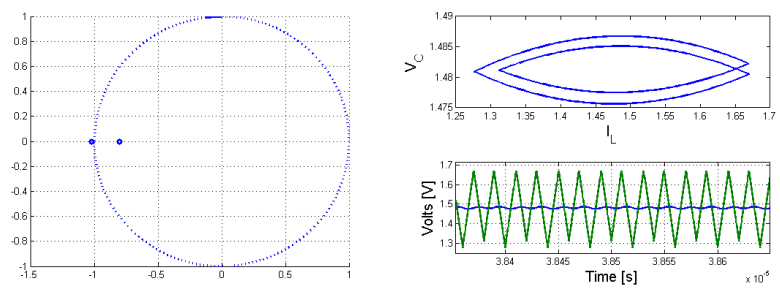

Figure 2. Eigenvalues obtained from Jacobian matrix and associated converter waveform results. $C=100 \mathrm{nF}, L=40 \mathrm{nH}, R=1 \quad, f_{s}=50 \mathrm{MHz}, k_{p}=26.5, V_{g}=3$ $\mathrm{V}, V_{\text {ref }}=1.5 \mathrm{~V}$

$\frac{\delta P}{\delta d_{n}}=\phi_{2} \Delta \dot{x}$ where $\Delta \dot{x}=\dot{x}^{*-} \quad \dot{x}^{*+}$ and $\dot{x}^{* \pm}$ corresponds respectively to the derivative, in steady-state, before and after the switching instant.

$\frac{\delta \sigma}{\delta d_{n}} \quad=\mathbf{K} \dot{x}^{*-} \quad m$ where $m$ is the slope of the PWM ramp $h(t)$ and $\mathbf{K} \dot{x}^{*-}$ is the derivative of the control signal just before the switching instant.

$\frac{\delta \sigma}{\delta x_{n}} \quad=\mathbf{K} \phi_{1}$ is the effect of a perturbation upon the switching surface.

By solving equation (5), the eigenvalues $(\lambda)$ of the Jacobian $D P$ are obtained.

$$
\operatorname{det}(D P \quad \lambda I)=0
$$

When one of the eigenvalues crosses the unit circle at the point $(-1,0)$ in the complex plane, fast-scale (period doubling or ip) bifurcation is exhibited, as observed in Fig. 2.

\section{DISCRETE-TIME MAPS APPROXIMATION AND RIPPLE-BASED INDEX DERIVATION}

The previous section has focused on demonstrating how fast-scale instabilities can be predicted through discrete-time maps. Nevertheless, it is also apparent that this approach involves sophisticated analysis that might not be of practical engineering use, lacking a circuit interpretation. In this section, such discrete-maps are simplified by using equations obtained from circuit theory in order to obtain a simple closed-form expression for predicting fast-scale instabilities.

Previous works [4], have also addressed the issue of obtaining a closed expression, but they do not consider simplifications and the derived expressions are still complicated thus making difficult their interpretation and their use to derive design criteria.

With this objective, the state transition $\phi_{i}(t)$ is simplified. Observe that this transition matrix corresponds to the smallsignal gain relating the final state conditions $\left(x_{i}\left(t_{i}\right)\right)$ to their initial conditions $\left(x_{i}\left(t_{0}\right)\right)$ in each time interval. From circuit theory it is possible to derive both state variables time-domain expressions:

$$
\begin{gathered}
v_{C}(t)=v_{C}\left(t_{0}\right)+\frac{1}{C} \int\left(i_{L}(t) \quad \frac{v_{C}(t)}{R}\right) \mathrm{d} t \\
i_{L}(t)=i_{L}\left(t_{0}\right)+\frac{1}{L} \int\left(\begin{array}{ll}
V_{g} & \left.v_{C}(t)\right) \mathrm{d} t
\end{array}\right.
\end{gathered}
$$


Note that from these expressions it is possible to obtain each coefficient of the transition matrices derived from initial conditions.

For the particular case of a buck converter, and considering the main terms of each state variable, the state transition matrix could be approximated as:

$$
\tilde{\phi}_{i}(t)=\left(\begin{array}{cccc}
1 & \frac{t_{i}}{R C} & \frac{t_{i}}{C} & \frac{t_{i}^{2}}{2 R C} \\
& \frac{-t_{i}}{L} & 1 & \frac{t_{i}^{2}}{2 L C}
\end{array}\right)
$$

If each term of the Jacobian matrix (Eq. 4) is reviewed according to the previous simplifications:

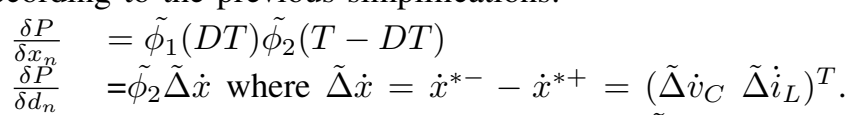
Taking into account circuit equations $\tilde{\Delta} \dot{v}_{C}$ could be considered almost zero and $\tilde{\Delta} i_{L}$ could be simplified as:

$$
\dot{i}_{L}^{*-}=\frac{V_{g} \quad v_{C}}{L} \quad \dot{i}_{L}^{*+}=\frac{v_{C}}{L}
$$

Therefore,

$$
\tilde{\Delta} \dot{i}_{L}=\frac{V_{g}}{L}
$$

$\frac{\delta \sigma}{\delta d_{n}}=k_{p} v_{C}^{\prime}(D T) \quad \frac{V_{m}}{T}$ by using a control based on voltage-feedback with proportional gain $k_{p}$ (Eq. 3).

$\frac{\delta \sigma}{\delta x_{n}}=\mathbf{K} \tilde{\phi}_{1}$

Finally by using the same expression as in (4), but based on the simplified terms, we can approximate the exact discretemap. The solution of the eigenvalues inequality equation (5), can accordingly be reformulated as:

$$
\lambda^{2} \operatorname{det}(D P) \quad \lambda \operatorname{tr}(D P)+1=0
$$

For the particular case of $\lambda-1$, a closed form expression for predicting fast-scale can be written as:

$$
\frac{\delta \sigma}{\delta d_{n}} \quad \frac{k_{p} V_{g}}{4 L C f_{s}} v
$$

where,

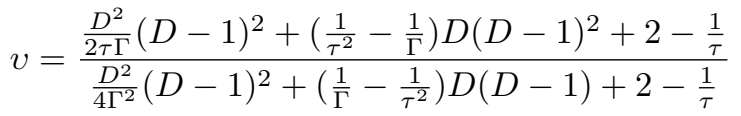

in which $\tau=\frac{1}{\omega_{c} T}$ where $\omega_{c}=(R C)^{-} 1$ and $\Gamma=\frac{1}{\left(\omega_{0} T\right)^{2}}$ where $\omega_{0}=(L C)^{-1}$ (the natural frequency of the second order filter of buck converter).

Up to now, a simplified closed form expression has been derived from the discrete-time map, but such expression does not provide direct design criteria. Analyzing the expression, as presented in the annex IV, demonstrates that the derivative at the switching instant can be obtained from the measure of the ripple $\Delta v_{c}$ of the converter.

$$
\Delta v_{C}=v_{C}^{\prime}(D T) \frac{T}{4}
$$

where $\Delta v_{C}$ can be approximated as, according to [12]:

$$
\Delta v_{C}=V_{g} \frac{D(1 \quad D)}{8 L C f_{s}^{2}}
$$

Re-arranging the expression describing period-doubling condition (8) according to the previous results, it is possible to obtain an expression where ripple appears explicitly:

$$
\rho=\frac{k_{p}}{V_{m}} \Delta v_{C} \quad \frac{k_{p}}{V_{m}} \frac{V_{g}}{8 L C f_{s}^{2}} \frac{v}{2} \quad \frac{1}{4}
$$

It is important to note the dependency of both sides of Eq. 12 to the same parameters with the same dependency except of duty cycle, which indicates that the stability boundary is not shifted when any of such parameters are modified. To make results consistent with the ripple-based approach (Eq. 1) it is useful to isolate all parameters except for $\mathrm{D}$, in the same right-side of the equation (Eq.13), in order to find an invariable expression of $\rho_{\text {critical }}$.

$$
\frac{k_{p}}{V m} \frac{V_{g}}{8 L C f_{s}^{2}} D(1 \quad D) \quad \rho_{\text {critical }}=\frac{1}{2} \frac{D(1 \quad D)}{v 2 D(1 \quad D)}
$$

Unveiling explicitly the ripple component in the closed-form equation, even as an indirect indication of the derivative at the switching instant, along with the variation obtained for the critical value, allows a design perspective for predicting fast-scale period-doubling stability condition.

Besides, such close expression simplifies the complex design-space into three parameters: $D, \Gamma$ and $\tau$. The in uence of these parameters upon stability boundary has been explored as shown in Fig. 3. The results obtained are contrasted to those obtained from discrete-time maps, showing the accuracy of the ripple-based stability condition. Results show that approximated $\rho_{\text {critical }}$ is a good approximation except for low $\Gamma$, which is directly related to the switching frequency and the natural frequency as was mentioned before. Note that the deviation is less than $5 \%$ by reducing $\Gamma$ to 5 , which implies a very low ratio $(2)$ between switching frequency $\left(f_{s}\right)$ to the natural second order filter $\left(f_{o}\right)$.

\section{CONCLUSIONS}

This paper has obtained a closed expression for predicting fast-scale instability boundary from a discrete-time map approximation. Such expression validates the recently proposed ripple-based index, which has a design-oriented perspective thereby allowing to consider the effect of each parameter of the circuit on the stability in the design phase. The results in this paper show that such ripple in the modulator input is an indirect observer of the control signal slope at the switching instant. Further investigations should study the effect of other controllers, such as PID, on the fast-scale stability boundary from a ripple perspective validated thorough discrete-time maps in order to obtain a complete model for predicting fastscale instabilities.

\section{APPENDIX}

\section{Relation between derivative at switching instant and ripple}

The aim of this appendix is to demonstrate that the ripple $\rho$ could be used as an indirect index of the output voltage derivative at the switching instant $\left(v_{C}^{\prime}(D T)\right)$. This hypothesis can 


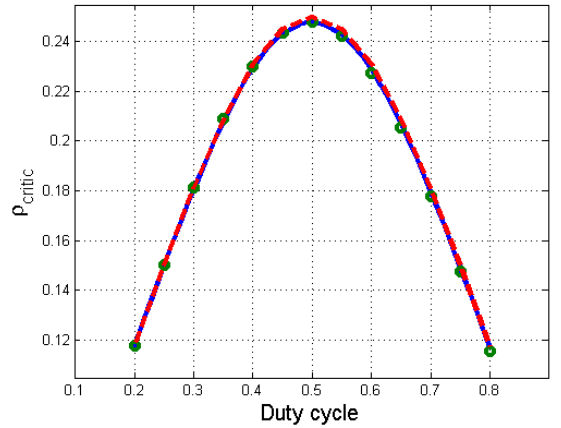

(a)

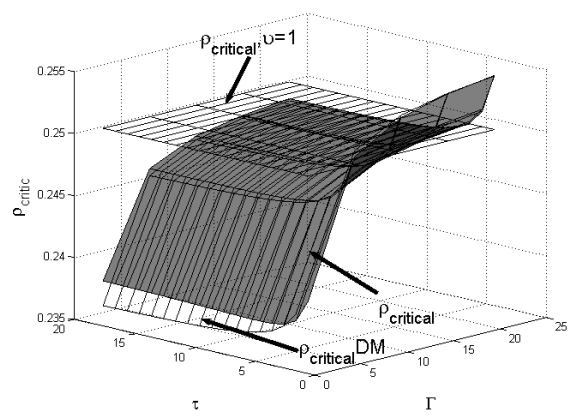

(b)

Figure 3. Stability boundary $\rho_{\text {critical }}$ exploration as function of (a) duty cycle (D) (b) $\tau$ and $\Gamma$. Results show data from exact (solid), and approximated $v=1 \rho_{\text {critical }}$ (dashed), and from exact discrete-map, DM (dots). $L=40$ $\mathrm{nH} C=100 \mathrm{nF} R=1 \quad f_{s}=50 \mathrm{Mhz} V_{g}=3 \mathrm{~V} V_{\text {ref }}=1.5 \mathrm{~V}$
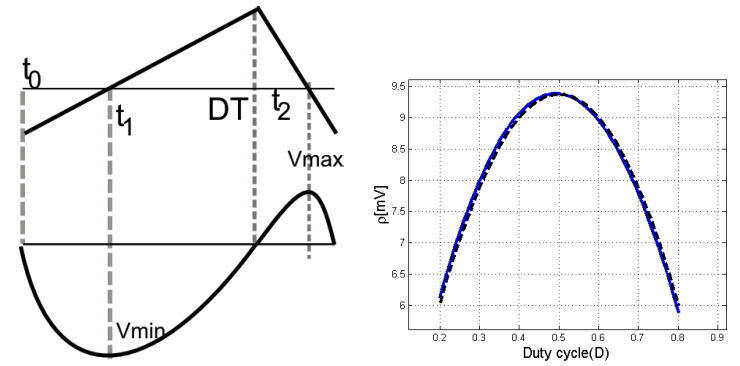

Figure 4. (a)Theoretical representation of current inductor (top) and output capacitor voltage (bottom). (b)Derivative at switching instant multiplied for a factor of $\frac{T}{4}$ (dashed) and measured peak-to-peak ripple (solid)

be validated by using the buck converter circuit expressions, according to Fig. 4.

$$
\begin{gathered}
v_{C}^{\prime}(D T)=\frac{V_{g}-v_{C}}{L C}\left(D T-t_{1}\right) \\
\Delta v_{C}=V_{\max }-V_{\min }=\left(t_{2}-t_{1}\right) \frac{V_{g}-V o}{L C}\left(D T-t_{1}\right)
\end{gathered}
$$

where $i_{C}$ is the capacitor current. Then:

$$
\Delta v_{C}=\left(t_{2}-t_{1}\right) v_{C}^{\prime}(D T)
$$

Where $t_{2}-t_{1}$ can be obtained from Fig. 4 by using circuit equations and considering low output ripple. Finally,

$$
\begin{array}{r}
t_{2}-t_{1}=\frac{T}{4} \\
\Delta v_{C}=v_{C}^{\prime}(D T) \frac{T}{4}
\end{array}
$$

Simulation showed in Fig. 4 validates the approximation.

\section{ACKNOWLEDGMENT}

This work is supported by FPU-grant of ministry of Science and Innovation of Spain (MICINN) and partial funding by project TEC2007-67988-C02-01 from the Spanish MICINN and EU FEDER funds.

\section{REFERENCES}

[1] R. Middlebrook and S. Cuk, "A general unified approach to modelling switching-converter power stages," in Power Electronics Specialists Conference, Cleveland, Ohio, June 8-10, 1976, Record, June 1976, pp. 18-34.

[2] D. C. Hamill and D. J. Jeffries, "Subharmonics and chaos in a controlled switched-mode power converter," IEEE Transactions on Circuits and Systems, vol. 35, no. 8, pp. 1059-1061, Aug. 1988.

[3] C. Tse, "Chaos from a buck switching regulator operating in discontinuous mode," Int. J. Circuit Theory Applicat., vol. 22, no. 6, pp. 263-278, Aug. 1994.

[4] M. di Bernardo, F. Garefalo, L. Glielmo, and F. Vasca, "Switchings, bifurcations, and chaos in DC/DC converters," Circuits and Systems I: Fundamental Theory and Applications, IEEE Transactions on, vol. 45, no. 2, pp. 133-141, feb 1998.

[5] K. Chakrabarty, G. Poddar, and S. Banerjee, "Bifurcation behavior of the buck converter," IEEE Transactions on Power Electronics, vol. 11, no. 3, pp. 439-447, May 1996.

[6] E. Fossas and G. Olivar, "Study of chaos in the buck converter," Circuits and Systems I: Fundamental Theory and Applications, IEEE Transactions on [see also Circuits and Systems I: Regular Papers, IEEE Transactions on], vol. 43, no. 1, pp. 13-25, Jan. 1996.

[7] A. El Aroudi, B. Robert, and L. Martinez-Salamero, "Bifurcation behavior of a three cell DC-DC buck converter," in 12th International Power Electronics and Motion Control Conference, Portoroz, Aug. 2006 , pp. 1994-2001.

[8] H. Iu and C. Tse, "Instability and bifurcation in parallel-connected buck converters under a master-slave current sharing scheme," in Power Electronics Specialists Conference, 2000. PESC 00. 2000 IEEE 31st Annual, vol. 2, 2000, pp. 708-713 vol.2.

[9] E. Alarcon, A. El-Aroudi, J. Martinez-Artega, G. Villar, F. Guinjoan, and A. Poveda, "Predicting fast-scale instabilities in switching power converters: a ripple-based unified perspective," in Circuits and Systems, 2006. ISCAS 2006. Proceedings. 2006 IEEE International Symposium on, May 2006.

[10] E. Rodriguez, G. Villar, F. Guinjoan, A. Poveda, A. El-Aroudi, and E. Alarcon, "General-purpose ripple-based fast-scale instability prediction in switching power regulators," in Circuits and Systems, 2007. ISCAS 2007. IEEE International Symposium on, New Orleans, LA, May 2007, pp. 2423-2426.

[11] A. El Aroudi, M. Debaat, L. Giral, G. Olivar, L. Benadero, and E. Toribio, "Bifurcations in dc-dc swithching converters: reivew of methods and applications," International Journal of Bifurcation and Chaos (IJBC), vol. 15, no. 5, pp. 1549-1578, Oct. 2005.

[12] R. W. Erickson and D. Maksimovic, Fundamentals of Power Electronics. Lluwer, 2001 